\title{
Compressible effects on a temporal direct numerical simulation
}

\author{
R. Sauvage and A. Kourta \\ Institut de Mécanique des Fluides de Toulouse \\ Avenue du Professeur Camille Soula \\ 31400 Toulouse \\ sauvage@imft.fr - kourta@imft.fr
}

\begin{abstract}
Direct numerical simulation of a temporal compressible mixing layer has been performed through the mixing layer transition at convective Mach number 0.4 with massively parallel computer. Finite volume method has been used to solve the full time-dependent, compressible Navier-Stokes equations without turbulence models. Numerical simulation initialized by the most amplified linear instability waves is then presented. $192^{3}$ mesh size is used to describe full scales. Roll-up and pairing process have developed and have been described, showing the highly two dimensional feature of the mixing layer. The influence of the phase between the two- and three-dimensional initially pertubated waves has been revealed .
\end{abstract}

\section{Introduction}

Renewed interest in high-speed flows from aircraft industry has stimulated fundamental research in the field of compressible turbulence. The development of propulsion systems based on supersonic combustion needs a best physical understanding of the compressible mixing layer. Study and understanding of the stability characteristics and transition to turbulence is of much theoretical and practical interest. The compressible mixing layer is a basic flow where mixing between two streams plays a predominant role for the aero-engine efficiency. It is now well established that compressibility can directly influence the performance of supersonic combustion chamber.

There are many experimental evidences of compressibility effects in mixing layers since the reduced turbulent shear layer growth with increased Mach number is one of the primary effects. This global effect has been observed by many authors (Papamoschou and Roshko 1988) (Elliott and Samimy 1990) (Papamoschou 1989), but no convincing explanation has been given. This growth rate reduction causes the stabilization of the flow in supersonic regime (Sarkar 1995).Thus, it was also observed (Clemens and Mungal 1992) (Lele 1994) that the large-scale organised structures play different role as Mach number increases. The roll-up and the pairing mechanisms exhibit similar features to the incompressible case if the Mach number is low whereas the scenario is quite different at higher Mach numbers $\left(M_{c}>0.6\right)$.

To characacterize the compressibility effects a convective Mach number $M_{c}$ has been introduced by Papamoschou\&Roshko (Papamoschou and Roshko 1988) based on previous Bogdanoff work (Bogdanoff 1983). Recent experiments (Clemens and Mungal 1995) revealed changes in turbulence statistics and typical eddy structure which becomes strongly three dimensional for convective Mach number greater than 0.6 .

The linear stability analysis has also developed (Ragab and Wu 1989) (Zhuang, Kubota and Dimotakis 1991) (Sandham and Reynolds 1990). Temporal and spatial stability problems have been solved and have shown that the most amplified disturbances remain two dimensional for convective Mach number up to 0.6. Several direct numerical simulation (Sandham and Reynolds 
1991) (Luo and Sandham 1994) (Vreman, Sandham and Luo 1996) have been investigated for different convective Mach numbers in two and three dimensional cases. Mixing transition to smallscale turbulence and the different stages of vortex formations have been analyzed at low Reynolds number (between 50 and 400).

Notwithstanding the low Reynolds number limitations, direct numerical simulation provide insight and guidance for the development of turbulence models. Few models exist in compressible flows (Zeman 1990) (Zeman 1991) (Lejeune and Kourta 1996) and they don't correctly describe physics of turbulence anisotropy and three dimensional effects in high Mach number unsteady flows, typical characteristics of the compressibility. Solving all the relevant scales of motion contribute to the fundamental understanding of the transition to turbulence mechanisms in compressible shear layers.

Because of the high demand on computational resources for a three dimensional spatially mixing layer simulation, the temporal mixing layer is considered, which qualitatively exhibits the same physical phenomena as the spatial case. This computational domain can be interpreted as 'a window' over the mixing layer that moves with the relative center plane velocity in the streamwise direction . Current simulations have been performed on massively parallel computer. This computing way allows to increase spatial resolution and to simulate higher Reynolds number flows. In this study, we focus on the transition process in temporal compressible mixing layer of convective Mach number 0.4, which brings up incompressible feature such as the two dimensional spanwise rollers that arose from the Kelvin-Helmoltz instability, and the decreasing growth rate compressible effect.

\section{Numerical methodology}

In this study, the three dimensional, time-dependent compressible Navier-Stokes equations have been solved by a finite volume method. The numerical technique uses a second order time and space accurate Mac Cormack explicit scheme. The explicit character of the scheme makes easier algorithm implementation, which opens very interesting future prospects in resolution and computing time. Based on the Simple Program Multiple Data method, the code uses Parallel Virtual Machine message passing library. The processor task sharing has been carried out by 'domain decomposition'. Thus, the physical domain is split in sub-domains, each one is associated with one processor. The current simulation has been performed on a T3E massively parallel computer with 64 nodes. So as to describe different Mach number cases (0.4 to 1.6), the Mac Cormack scheme is particularly interesting to capture strong discontinuities (shocklets) at high Mach number. The previous studies (Vreman et al. 1996) have used different numerical schemes to analyse separately low and high Mach numbers. In our case, we would like to use the same scheme both in low and high Mach number range to better describe the Mach number effects. This scheme could be also used to simulate hypersonic flow with the only limitation of perfect gas hypothesis. Moreover, it is very flexible and allows a very easy boundary conditions integration without using extrapolation or filtering, necessary condition for higher accurate numerical scheme.

Streamwise (x) and spanwise (z) boundary conditions are treated as periodic. Whereas the boundaries in the normal direction are treated as free-slip walls in previous works (Vreman et al. 1996) (Ragab, Shaw-Ching and Sreedhar 1992), the non-reflecting characteristic boundary conditions developed by (Thompson 1987) and modified by (Poinsot and Lele 1992) for viscous flow are imposed in this work so as to reduce confining effect. Uniform Cartesian grid is used in spanwise 
and streamwise directions whereas a refined mesh in the center of the layer is defined with a sinus hyperbolic law.

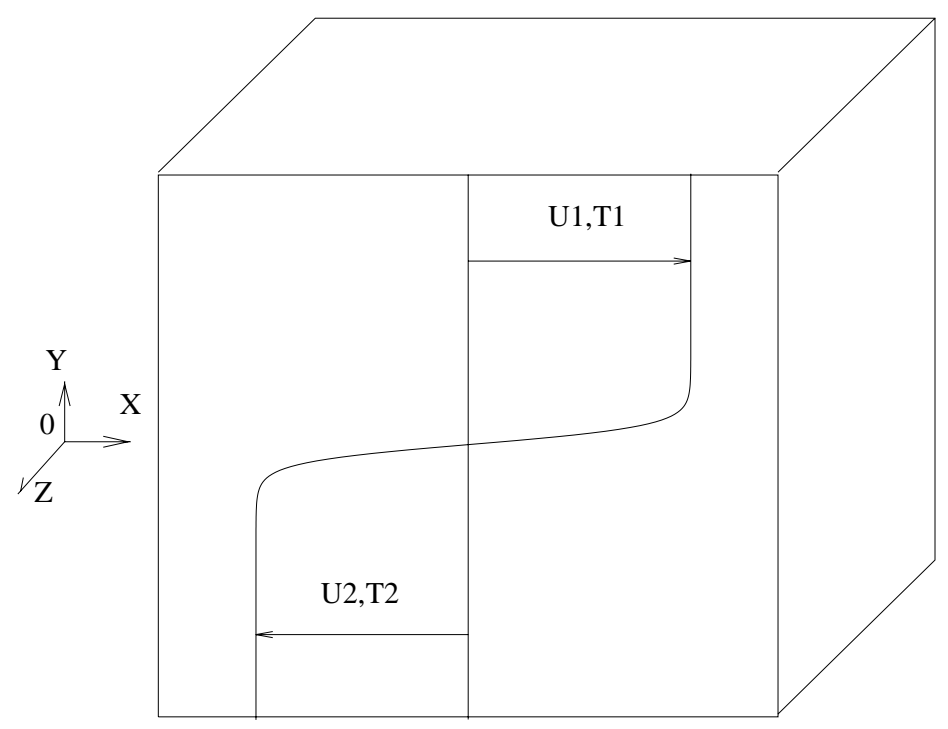

Figure 1: Computational domain of the mixing layer

The convective Mach number $M_{c} 0.4$ is chosen because in this case, incompressible and weak compressible feature would be present in the mixing layer. $M_{c}$ is defined as $M_{c}=\left(U_{1}-U_{2}\right) /\left(c_{1}+\right.$ $\left.c_{2}\right)$ where $U_{1}$ and $U_{2}$ are the free-stream velocities and $c_{1}$ and $c_{2}$ are the corresponding sound speeds. Thus, the temporally evolving shear layer simulations are initialized with an hyperbolic tangent mean streamwise velocity profile and zero mean transverse and spanwise velocities. The temperature profile follows the Busemann-Crocco law (Sandham and Reynolds 1990) assuming equality of the mean temperature in the two streams. The mean velocity and temperature profiles are as follows:

$$
\begin{gathered}
\frac{\bar{U}}{U_{1}}=\tanh \left(\frac{2 y}{\delta_{\omega 0}}\right) \\
\frac{\bar{T}}{T_{1}}=1+M_{c} \frac{\gamma-1}{2}\left(U_{1}+\bar{U}\right)\left(\bar{U}-U_{2}\right)
\end{gathered}
$$

All the thermodynamics properties are initialized to uniform values. The initial Reynolds number based on the velocity difference across the layer and the initial vorticity thickness is equal to 200. Its choice isn't dependent on the numerical scheme. However, its limitation is linked to the mesh. The vorticity thickness is defined as $\delta_{\omega}=\left(U_{1}-U_{2}\right) /|d \bar{u} / d y|_{\max }$. As the vorticity thickness increases during the simulation, the Reynolds number also increases in the time and could reach much higher than 1000 . 
The initial flow field is superimposed with two and three dimensional perturbation modes obtained from linear stability analysis. We have developed a code solving the inviscid compressible equation of linear stability to calculate amplified mode and their associated eighenfunctions $\widehat{\Phi}$. Results were validated with different works (Sandham and Reynolds 1991). The streamwise and the spanwise fundamental wavelengths $\left(\lambda_{x}\right.$ and $\left.\lambda_{z}\right)$ of the simulation are chosen to be the most instable wavelengths from linear stability theory. The streamwise and spanwise associated wavenumbers $\left(k_{x}\right.$ and $k_{z}$ ) are introduced by $k_{x}=2 \pi / \lambda_{x}$ and $k_{z}=2 \pi / \lambda_{x}$. To specify the initial conditions, we define a single mode as

$$
(\alpha, \beta)=\left(\frac{k_{x} \lambda_{x}}{2 \pi}, \frac{k_{z} \lambda_{z}}{2 \pi}\right)
$$

where $(\alpha, \beta)$ are the integer wavenumbers. Two dimensional perturbations are defined by $(2,0)$ and $(1,0)$, the most amplified mode and its subharmonic, respectively. The three dimensional perturbations are introduced by equal and opposite oblique modes $(2,2),(2,-2),(1,1),(1,-1)$. The initial condition for such a combination of waves can be written as

$$
\Phi^{\prime} \sim a_{\alpha} \operatorname{Re}\{\widehat{\Phi}(\alpha, 0) \exp (i(\alpha x+\phi))\}+b_{\alpha \beta} \operatorname{Re}\{\widehat{\Phi}(\alpha, \beta) \exp (i(\alpha x \pm \beta z))\}
$$

for $u^{\prime}, v^{\prime}, w^{\prime}, \rho^{\prime}$ and $T^{\prime}$.The amplitudes of perturbations $a_{\alpha}$ and $b_{\alpha \beta}$ have been chosen as $a_{2}=0.05$, $a_{1}=0.025, b_{2 \pm 2}=b_{1 \pm 1}=0.025$. $\phi$ is the phase difference between the two dimensional wave and the pair of oblique waves. For this study, $\phi$ is equal to 0 . Its important effect will discuss below.

In accordance with these perturbations, the computational domain size is two times the fundamental wavelength associated to the most amplified mode, i.e. $L_{x}=L_{z}=15.32 \delta_{\omega 0}$. For this study, the temporal and spatial adimensionnal increments equal the following values: $\Delta t=0.0087$ and $\Delta x=0.0798$. The time step has to satisfy the Courant-Friedrichs-Levy condition $(<1)$. It is taken to 0.9 .

\section{Results and discussion}

The temporal code was validated against typical two dimensional tests on boundary conditions and against transition results of Sandham\&Reynolds. The mesh size uses a $192 \times 192 \times 192$ grid. This mesh is enought fine to compute all scales. 50 T3E massively parallel computer hours have been needed to simulate 14 wavelenghts. During the simulation, the two dimensional energy spectrum $E\left(k_{x}, k_{z}\right)$ (not shown) has been computed to ensure adequate resolution. It was defined by

$$
E\left(k_{x}, k_{z}\right)=\int_{-L y / 2}^{L y / 2} \widetilde{u}_{i}\left(k_{x}, k_{z}\right) \widetilde{u}_{i}^{\dagger}\left(k_{x}, k_{z}\right) d y
$$

where †indicates a complex conjugate. 

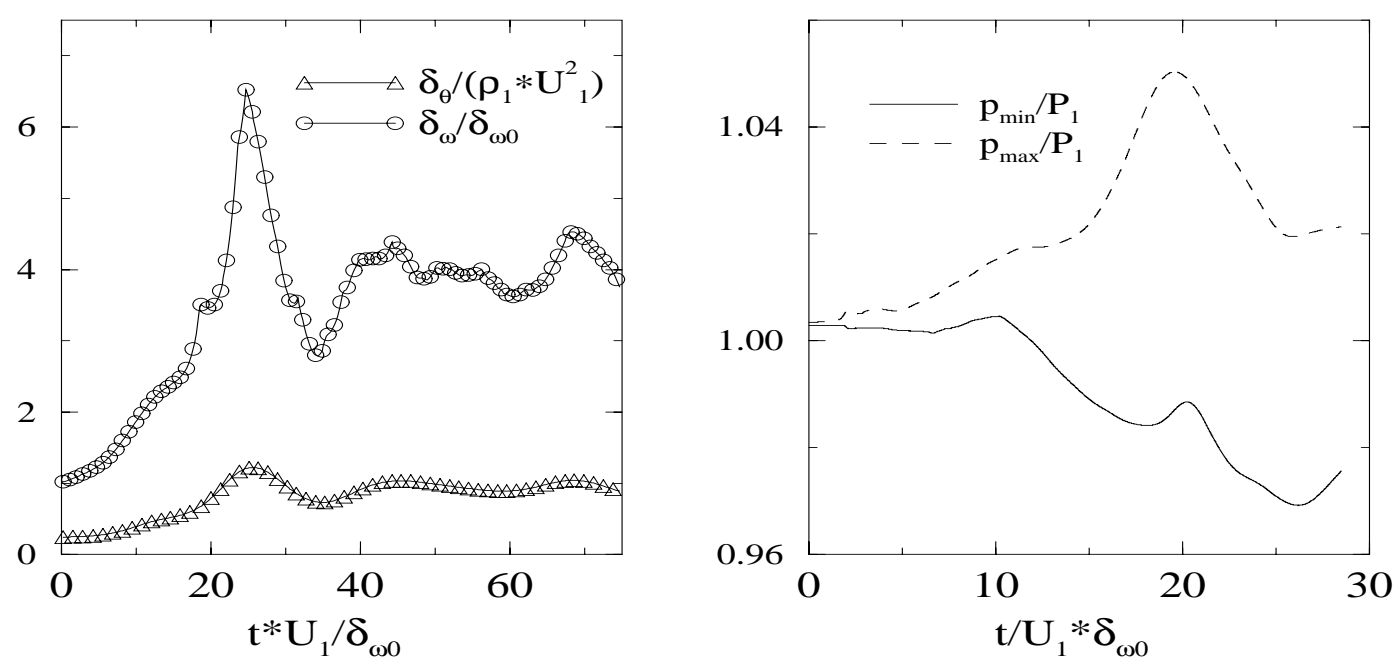

Figure 2: Temporal evolution of a) the vorticity and momentum thicknesses; b) the minimum and maximum pressure.

A large decrease in energy at high wavelenghts indicates that the flow is fully resolved.

The development of the vorticity and momentum thicknesses is shown in figure 2-a. At the beginning, both thicknesses increase quasi-linearly until $t=18$. The shear layer extends by diffusion. In this stage, perturbated by initial two dimensional instability, the centerplane vorticity area begins to undulate, shown by the contours of spanwise velocity component in one (x,y) plane (top of figure 3 -a) at $\mathrm{t}=4.5$. In the corresponding pressure field (bottom), pressure depression and overpressure spots appear in the flow across the shear layer. Maxima and minima pressure history plotted on figure 2-b allows to describe the different stages of the temporal development of the compressible mixing layer. For instance, strong eddies existence depends on any large local pressure depression in the flow. Between $\mathrm{t}=10.5$ and $\mathrm{t}=18$., pressure minimum decreases quickly. Two spanwise well-developed rollers come together bound by braid regions shown in the top figure 3 - $\mathrm{b}$ at $\mathrm{t}=13.17$. At the bottom, pressure contours reveal the eddies appearance.

The now familiar pairing could henceforth begin to process. The vorticity thickness highly increases, the two structures roll up and corotate, concentrating the total vorticity into the new paired eddy. This can be seen in paired figures 3 -c for $t=23.85$. It is corresponding to the second pressure minimum decrease shown in figure 2-b. This process involves spanwise vorticity decrease in the surviving braid region. As the cores of the two spanwise eddies merge into a new one, spiral arms of weaker spanwise vorticity are injected away from the paired structure. These arms stretch out arising from the shear motion and have drawn back into the surviving braid region developing a hook caracteristic (as can be seen in the top figure d) following the influence of the paired structure periodic images.

After the pairing, the spiral arms roll up around the paired structure decreasing vorticity quantities into the braid region and are injected away again, bringing spanwise vorticity back into the braid area an other time. This process repeats itself until "oversaturation" as it can be seen in figure 4 at $\mathrm{t}=75$. This concept has been introduced and described by (Moser and Rogers 1993) for the incompressible case. It's caused by the advection of vorticity into the braid region. A the end of the simulation, the concentration of spanwise vorticity in the last structure involves the eddy lengthscale close to the box one. As the Kelvin-Helmoltz rollers became increasingly distorted and 
a)
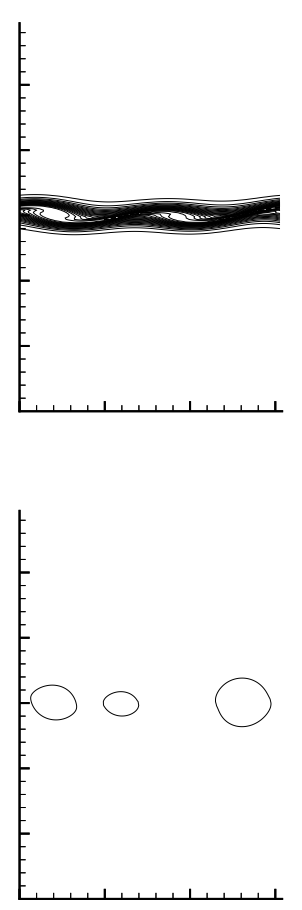

b)
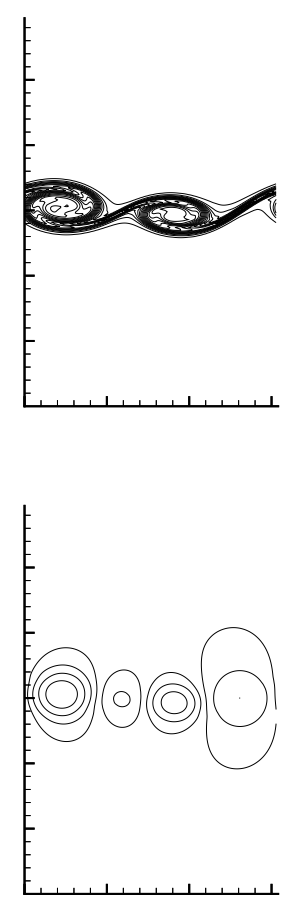

c)
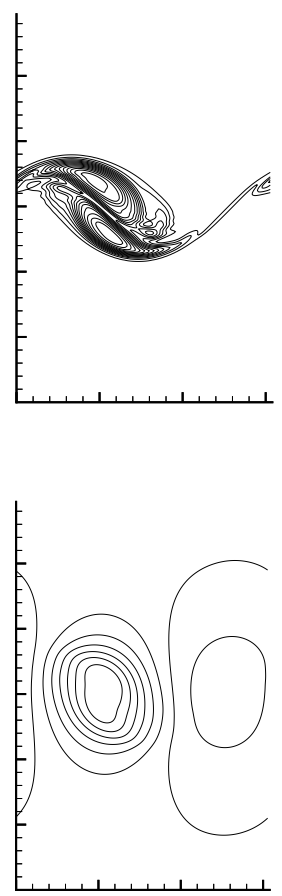

d)
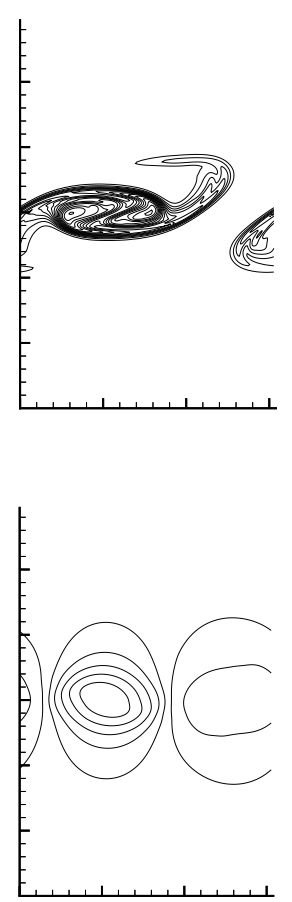

Figure 3: Contours of spanwise vorticity $\omega_{z}$ (top) and pressure (bottom) in the $z=L_{z} / 2$ plane at a) $\mathrm{t}=4.5$; b) $\mathrm{t}=13.17$; c) $\mathrm{t}=23.85$; d) $\mathrm{t}=36.6$. The contour increment is 0.05 for $\omega_{z}$ and 0.1 for the pressure.

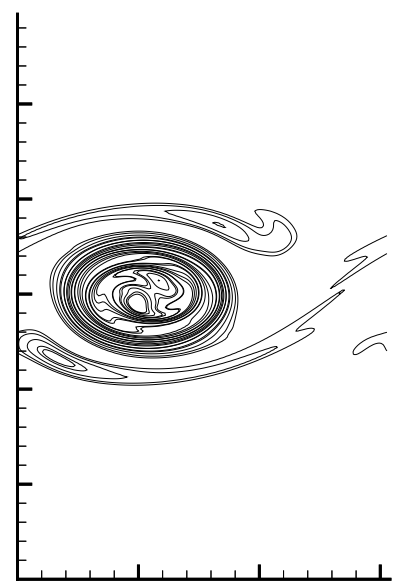

Figure 4: Contours of the spanwise vorticity when oversaturation is established at $t=75$. 
gradually lost their organised structure in the

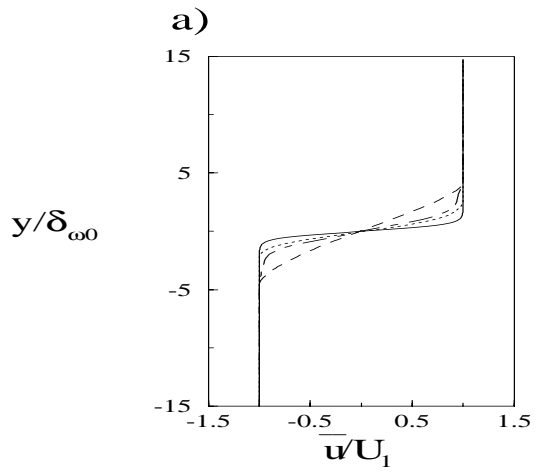

d)

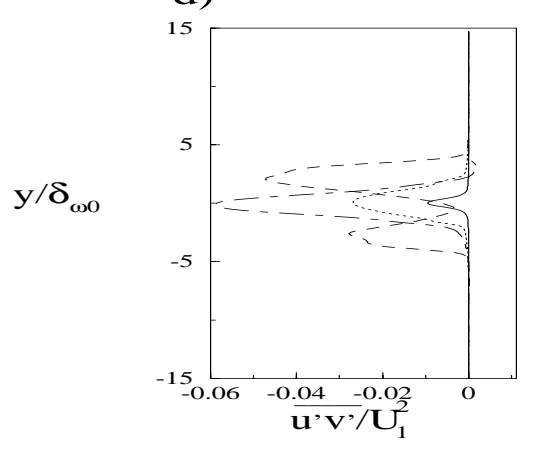

b)

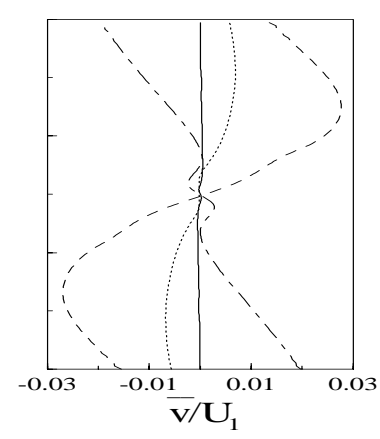

e)

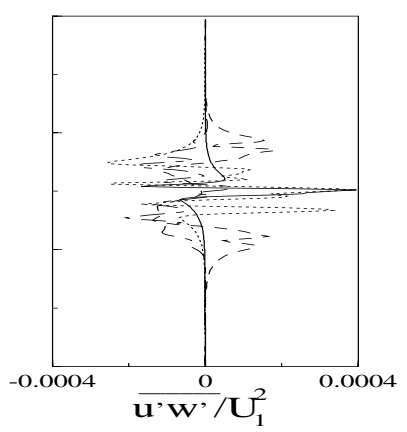

c)

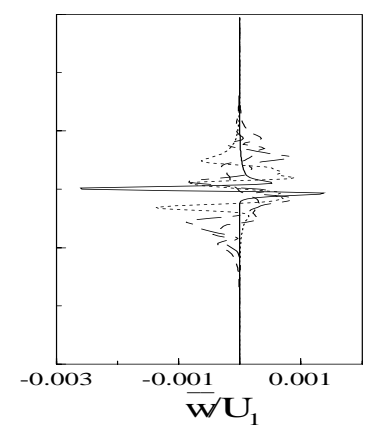

f)

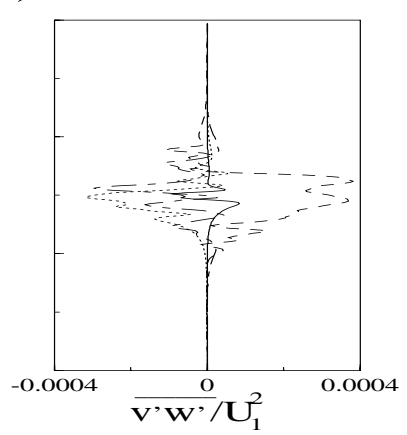

Figure 5: Mean profile at $\mathrm{t}=4.5$ (solid curve); $\mathrm{t}=13.17$ (dotted); $\mathrm{t}=23.85$ (dashed); $\mathrm{t}=36.6$ (dotdashed).

incompressible case, the three dimensional vizualisation of surfaces of constant vorticity show a highly two dimensional structure. This one varies sinusoidally in the spanwise direction. The initial three dimensional perturbations have not been enought amplified to destabilize spanwise direction of the flow. This result is similar with Sandham \& Reynolds' ones, which argue about the real influence of initial conditions, although this previous work used one two dimensional mode $(1,0)$ and a pair of equal and opposite oblique waves $(1, \pm 1)$. Thus, the role of the difference of phase $\phi$ between the two dimensional waves and the pair of obliques waves is quite important as the secondary mode depends on its choice. In our study, we have taken $\phi=0$ which excites the "bulging" mode of instability. The developped structure become strongly two dimensional in spite of the initial three dimensional perturbations. The terminology is taken from (Pierrehumbert and Widnall 1983). Moreover, the case $\phi=\pi / 2$, named the "translative" mode and studied by (Sandham and Reynolds 1991), leads to a more amplified mode, giving a final structure which oscillates in the spanwise direction. It constitues the mean secondary instability found in three dimensional incompressible mixing layer. Since the computational domain is finite in the homogeneous directions, it is very difficult to achieve self-similarity with direct numerical simulation initialized by small disturbances. Nevertheless, the growth rate $d \delta_{\omega} / d t$ can be approximated on the figure 2-a when pairing can no longer occur at the end of the simulation, giving 0.112 between $t=35$. and $\mathrm{t}=43.6$, which is lower compared with experimental incompressible growth rate 0.181 (Brown and Roshko 1974), showing compressible effect.

Finally, the evolution of the Reynolds stress components $\overline{u^{\prime} v^{\prime}}, \overline{u^{\prime} w^{\prime}}$ and $\overline{v^{\prime} w^{\prime}}$ and mean speed during the transition is shown in figure 5. These quantities are averaged over $\mathrm{x}$ and $\mathrm{z}$. The bar sign 
indicates Reynolds averaging and the prime indicates fluctuations based on Reynolds averaging. Up the pairing process, streamwise and normal mean velocity profiles (a,b) change gradually, the developped eddies injecting fluid away. After this stage,the roll-up around the paired structure (explained above) causes the re-entering motion of fluid as can be seen on the dot-dashed curve of $\bar{v}$ profile. The $\overline{u^{\prime} v^{\prime}}$ profile is dominated by single peak in the central layer as long as the two structures have not collapsed, characterized by double peak appearance (dashed curve in figure d). As can be seen in figures c,e,f, the weak amplitudes of spanwise mean velocity and $\overline{u^{\prime} w^{\prime}}$ and $\overline{v^{\prime} w^{\prime}}$ stress Reynolds components profiles argue about the two dimensional feature of the mixing layer during transition process.

\section{Conclusion}

A direct numerical simulation of a temporal weakly compressible mixing layer at convective Mach number has been studied. Parallel algorithm has been implemented allowing high resolution. The simulation has been excited by two dimensional waves (two modes) and a pair of oblique waves. Two dimensional spanwise rollers developed, collapsed and amalgamed. Roll-up and pairing mecanisms have been qualitatively and quantitatively described. The process of the development and the pairing is very close to the incompressible case dominated by the Kelvin-Helmoltz instability studied by Moser\&Roger. The explanation of the highly two dimensional structure of the simulation proceeds from the choice of the phase between the two different tips of the initial perturbations, which shows the real influence of initial conditions. This result is very similar with the Sandham\&Reynolds works on compressible mixing layer, in which just one mode is used. Even if it is weak at 0.4 convective Mach number, the compressibility effect is mainly obtained on the reduction of the growth rate of the mixing layer compared to the corresponding incompressible case.

Acknowledgement 1 This work was performed at Institut de Mecanique des Fluides de Toulouse. Computational facilities were provided by IDRIS at Orsay (France). This support is gratefully acknowledged.

\section{References}

Bogdanoff, D.: 1983, Compressibility effects in turbulent shear layers, AIAA Journal 21, 926-927.

Brown, G. L. and Roshko, A.: 1974, On density effects and large scale structure in turbulent mixing layers, J. Fluid Mech. 64, 775-816.

Clemens, N. and Mungal, M. G.: 1992, Two- and three-dimensional effects in the supersonic mixing layer, AIAA Journal 30, 973-981.

Clemens, N. and Mungal, M. G.: 1995, Large-scale structure and entrainment in the supersonic mixing layer, J. Fluid Mech. 284, 171-216.

Elliott, G. S. and Samimy, M.: 1990, Compressibility effects in free shear layers, Phys. Fluids A $(2), 1231-1240$.

Lejeune, C. and Kourta, A.: 1996, Modelling of high speed turbulent flows, AIAA Paper 96-2041. 
Lele, S. K.: 1994, Compressibility effects on turbulence, Ann. Rev. Fluid Mech. 26, 211-254.

Luo, K. H. and Sandham, N. D.: 1994, On the Formation of Small Scales in a Compressible Mixing Layer, In Direct and Large-Eddy Simulation, Kluwer.

Moser, R. D. and Rogers, M.: 1993, The three-dimensional evolution of a plane mixing layer: Pairing and transition to turbulence, J. Fluid Mech. 247, 275-320.

Papamoschou, D.: 1989, Structure of the compressible turbulent shear layer, AIAA Paper 890126.

Papamoschou, D. and Roshko, A.: 1988, The compressible turbulent mixing layer: An experimental study, J. Fluid Mech. 197, 453-477.

Pierrehumbert, R. T. and Widnall, S. E.: 1983, The two- and three-dimensional instabilities of a spatially periodic shear layer, J. Fluid Mech. 114, 59-62.

Poinsot, T. J. and Lele, S. K.: 1992, Boundary conditions for direct simulations of compressible viscous reacting flows, J. Comp. Physics 101, 104-129.

Ragab, A. and Wu, J. L.: 1989, Linear instabilities in two-dimensional compressible mixing layers, Phys. Fluids A(6), 957-966.

Ragab, S. A., Shaw-Ching, S. and Sreedhar, M.: 1992, An investigation of finite-difference methods for large-eddy simulation of a mixing layer, AIAA Paper 0554 .

Sandham, N. D. and Reynolds, W. C.: 1990, Compressible mixing layer: Linear theory and direct simulation, AIAA Journal 28, 618-624.

Sandham, N. D. and Reynolds, W. C.: 1991, Three-dimensional simulations of large eddies in the compressible mixing layer, J. Fluid Mech. 224, 133-158.

Sarkar, S.: 1995, The stabilizing effect of compressibility in turbulent shear flow, J. Fluid Mech. 282, $163-186$.

Thompson, K. W.: 1987, Time dependent boundary conditions for hyperbolic systems, J. Comp. Phys. 68, 1-24.

Vreman, A. W., Sandham, N. D. and Luo, K. H.: 1996, Compressible mixing layer growth rate and turbulence characteristics, J. Fluid Mech. 320, 235-258.

Zeman, O.: 1990, Dilatation dissipation: The concept and application in modeling compressible mixing layers, Phys. Fluids A(2), 178-188.

Zeman, O.: 1991, On the decay of compressible isotropic turbulence, Phys. Fluids A(3), 951-955.

Zhuang, M., Kubota, T. and Dimotakis, P. E.: 1991, Instability of inviscid, compressible free shear layers, Phys. Fluids 28(10), 1728-1733. 